\title{
DETERMINACY WITH COMPLICATED STRATEGIES
}

\author{
ALEXANDER S. KECHRIS
}

\begin{abstract}
For any class of functions $\mathfrak{F}$ from $\mathbf{R}$ into $\mathbf{R}, A D(\mathfrak{F})$ is the assertion that in every two person game on integers one of the two players has a winning strategy in the class $\mathfrak{F}$. It is shown, in $Z F+D C+V=L(\mathbf{R})$, that for any $\mathfrak{F}$ of cardinality $\leq 2^{\boldsymbol{N}_{0}}$ (i.e. any $\mathfrak{F}$ which is a surjective image of $\mathbf{R}$ ) $\mathrm{AD}(\mathfrak{F})$ implies $\mathrm{AD}$ (the Axiom of Determinacy).
\end{abstract}

1. Statement of the results. Let $\omega=\{0,1,2, \ldots\}$ be the set of natural numbers and let $\mathbf{R}=\omega^{\omega}$ be the set of all infinite sequences from $\omega$, called for simplicity reals in the following. Given any set $A \subseteq \mathbf{R} \times \mathbf{R}$ we associate with $A$ the following infinite game also denoted by $A$ : In a run of the game players I and II choose alternatively natural numbers $x(0), y(0), x(1), y(1), \ldots$

$$
\begin{aligned}
& \text { I } x(0) \quad x(1) \\
& \text { II } \quad y(0) \quad y(1)
\end{aligned}
$$

and player I wins this run if $(x, y) \in A$; otherwise II wins. A strategy for player I is a map $\sigma: \omega^{<\omega} \rightarrow \omega$, where $\omega^{<\omega}=$ the set of all finite sequences from $\omega$. If II plays $(y(0), y(1), \ldots)$ then I follows the strategy $\sigma$ if he responds by playing $x(0), x(1), \ldots$, where $x(n)=\sigma(y \mid n)$, with $y \mid n=(y(0), \ldots, y(n-1))$. The strategy $\sigma$ is winning for player I in the game $A$, if for all $y \in \mathbf{R}$ when II plays $y$ and I plays $x$ following $\sigma$ then $(x, y) \in A$. Similarly we define the concept of a strategy $\tau$ for player II and what it means to be winning for him. The set $A$ is determined if either player I or II has a winning strategy for the game $A$. The Axiom of Determinacy (AD) is the assertion that all sets $A \subseteq \mathbf{R} \times \mathbf{R}$ are determined.

If $\sigma$ is a strategy for player I, then $\sigma$ can be viewed as a function $\sigma^{*}: \mathbf{R} \rightarrow \mathbf{R}$ given by $\sigma^{*}(y)=x$, where $x(n)=\sigma(y \mid n)$. Similarly if $\tau$ is a strategy for II we denote by $\tau^{*}$ the corresponding function. Thus $\mathrm{AD}$ can be rewritten as follows:

For all $A \subseteq \mathbf{R} \times \mathbf{R}$, either there is a strategy $\sigma^{*}$ such that $\forall y\left(\sigma^{*}(y), y\right) \in A$ or else there is a strategy $\tau^{*}$ such that $\forall x\left(x, \tau^{*}(x)\right) \notin A$.

The functions of the form $\sigma^{*}$ or $\tau^{*}$ are clearly continuous functions on $\mathbf{R}$ of a very special kind, i.e. Lipschitz. What if we consider instead "strategies" which are more complicated? To make this question precise let us give the following definition.

Received by the editors January 3, 1984.

1980 Mathematics Subject Classification. Primary 03E60, 03E15, 03E55; Secondary 03E45. 
Definition. Let $\mathfrak{F}$ be a collection of functions from $\mathbf{R}$ into $\mathbf{R}$. By $\mathrm{AD}(\mathfrak{F})$ we abbreviate the statement:

For all $A \subseteq \mathbf{R} \times \mathbf{R}$ either there is $f \in \mathfrak{F}$ with $\forall y(f(y), y) \in A$ or there is $g \in \mathfrak{F}$ with $\forall x(x, g(x)) \notin A$.

A geometric reformulation of $\mathrm{AD}(\mathfrak{F})$ is the following: If $P \subseteq \mathbf{R} \times \mathbf{R}$ is a relation and $h: \mathbf{R} \rightarrow \mathbf{R}$ is a function, then we say that $h$ uniformizes $P$ in the $y$-direction if $\forall x(x, h(x)) \in P$. We say that $h$ uniformizes $P$ in the $x$-direction if $\forall y(h(y), y) \in P$. Then $\mathrm{AD}(\mathfrak{F})$ asserts that for any $A \subseteq \mathbf{R} \times \mathbf{R}$ there is a function in $\mathfrak{F}$ which uniformizes $A$ in the $x$-direction or there is a function in $\mathfrak{F}$ which uniformizes $(\mathbf{R} \times \mathbf{R})-A$ in the $y$-direction.

Clearly $\mathrm{AD}$ implies $\mathrm{AD}(\mathfrak{F})$ for any $\mathfrak{F}$ containing the Lipschitz functions. Mycielski [7] has considered first the question of the strength of $\mathrm{AD}$ (Continuous). Later $\mathrm{H}$. Friedman [2] asked more generally about $\mathrm{AD}$ (Borel), $\mathrm{AD}$ (Projective), etc. A. Blass [1] proved that $\mathrm{AD}$ (Continuous) $\Rightarrow \mathrm{AD}$ and Kunen [6] strengthened it substantially by showing that $\mathrm{AD}$ (Borel) $\Rightarrow \mathrm{AD}$, and indeed that $\mathrm{AD}\left(\Delta_{2}^{1}\right) \Rightarrow \mathrm{AD}$. Some further results have been obtained in [3].

We prove in this paper an almost optimal generalization of these results, at least in the case $V=L(\mathbf{R})$, where $L(\mathbf{R})$ is the smallest inner model of $Z F$ containing $\mathbf{R}$. First let us say that a class $\mathfrak{F}$ of functions from $\mathbf{R}$ into $\mathbf{R}$ has cardinality $\leq 2^{\boldsymbol{\aleph}_{0}}$ if there is a surjection $p: \mathbf{R} \rightarrow \mathfrak{F}$. Then we have (assuming $\mathfrak{F} \supseteq$ Lipschitz)

THEOREM $(Z F+D C)$. (a) For any class of functions $\mathfrak{F}$ ( from $\mathbf{R}$ into $\mathbf{R})$ which has cardinality $\leq 2^{\aleph_{0}}, \mathrm{AD}(\mathfrak{F}) \Rightarrow L(\mathbf{R}) \vDash \mathrm{AD}$. In particular, if $V=L(\mathbf{R})$ and $\mathfrak{F}$ has cardinality $\leq 2^{\aleph_{0}}, \mathrm{AD}(\mathfrak{F}) \Rightarrow \mathrm{AD}$.

(b) If $\mathfrak{F}$ is a class of functions, $\mathfrak{F} \subseteq L(\mathbf{R})$ and $\mathfrak{F}$ has cardinality $\leq 2^{\aleph_{0}}, \mathrm{AD}(\mathfrak{F}) \Rightarrow$ AD.

COROLLARY. The following two theories are equiconsistent:

(i) AD.

(ii) There is a class $\mathfrak{F}$ of functions ( from $\mathbf{R}$ into $\mathbf{R}$ ) of cardinality $\leq 2^{\aleph_{0}}$ such that $\mathrm{AD}($ if $)$ holds.

Remarks. (1) Clearly if $\mathfrak{F}=$ the class of all continuous, $\Delta_{2}^{1}$, projective, etc. functions, then $\mathfrak{F} \subseteq \mathrm{L}(\mathbf{R})$ and $\mathfrak{F}$ has cardinality $\leq 2^{\aleph_{0}}$, so (b) is a strengthening of the earlier results, and provides a full answer to the question of $\mathrm{H}$. Friedman.

(2) If $\overline{\mathfrak{F}}=$ the class of all functions on $\mathbf{R}$, then the assumption that every $A \subseteq \mathbf{R} \times \mathbf{R}$ can be uniformized implies trivially $\operatorname{AD}(\overline{\mathfrak{F}})$, thus $\operatorname{AD}(\overline{\mathfrak{F}})$ is a weak hypothesis (equiconsistent with $Z F$ ).

(3) Our method of proof of (b) provides also simple proofs of some earlier results, such as $\mathrm{AD}($ Borel $) \Rightarrow \mathrm{AD}$. We explain this after giving the proof of the main theorem.

In conclusion, we would like to thank $H$. Becker for pointing out that the conditions on $\mathfrak{F}$ under which an original version of (a) was proved amount to just that $\mathfrak{F}$ has cardinality $\leq 2^{\aleph_{0}}$. 
2. Proofs. We first prove part (a) of the theorem. Our argument combines the ideas of [4 and 5], with which we have to assume familiarity. (The proof of (b), which contains the main new idea of this paper, is however self-contained modulo just the statement of part (a).)

Let $\mathfrak{F}$ be a class of functions of cardinality $\leq 2^{\aleph_{0}}$ such that $\operatorname{AD}(\mathfrak{F}$ ) holds (we assume of course throughout $Z F+D C)$. In order to prove that $L(\mathbf{R}) \vDash A D$ we will use the following result proved in [4]:

If for any $\lambda<\Theta$ there is a cardinal $\kappa>\lambda$ with the strong partition property $\kappa \rightarrow(\kappa)^{\kappa}$, then $L(\mathbf{R}) \vDash \mathrm{AD}$.

So it is enough to prove that $\forall \lambda<\Theta \exists \kappa>\left(\kappa \rightarrow(\kappa)^{\kappa}\right)$. Now in [5] it is indeed shown that

$$
\mathrm{AD} \Rightarrow \forall \lambda<\Theta \exists \kappa>\lambda\left(\kappa \rightarrow(\kappa)^{\kappa}\right) .
$$

Our main observation is that one can still prove

$$
\mathrm{AD}(\mathfrak{F}) \Rightarrow \forall \lambda<\Theta \exists \kappa>\lambda\left(\kappa \rightarrow(\kappa)^{\kappa}\right)
$$

provided $\mathfrak{F}$ has cardinality $\leq 2^{\aleph_{0}}$.

Indeed fix $\lambda<\Theta$ and let $\pi: \mathbf{R} \rightarrow \mathfrak{F}$ be a surjection. Let $A \subseteq \mathbf{R}^{3}$ be the relation that codes $\pi$, i.e.

$$
(x, y, z) \in A \Leftrightarrow \pi(x)(y)=z .
$$

Let now, using the terminology of [5], $\Gamma$ be a Spector pointclass closed under ${ }^{3} E$ such that $\lambda<\kappa=0(\Delta)$ ( = supremum of the ranks of prewellorderings in $\Delta$ ) and $A \in \Delta$. Now by [5, Theorem 1.1], if we knew that AD holds then we would have that $\kappa$ has the strong partition property. The following change in the proof of [5, Theorem 1.1] makes the same proof work under the weaker assumption that $\operatorname{AD}(\mathfrak{F})$ holds:

In p. 80 of [5] modify the definition of $\Sigma_{1}^{1}(\chi)$ formulas to the obvious definition of $\Sigma_{1}^{1}(\chi, \psi)$ formulas, where $\chi$ denotes now a partial function from $\mathbf{R} \times \mathbf{R}$ into $\omega$ and $\psi$ a partial function from $\mathbf{R} \times \mathbf{R} \times \mathbf{R}$ into $\omega$. Then let $\Sigma_{1}^{1}(\chi ; A)$ be $\Sigma_{1}^{1}\left(\chi ; \psi_{A}\right)$, where $\psi_{A}$ is the characteristic function of $A$. Replace now in the rest of the proof of [5, Theorem 1.1], $\Sigma_{1}^{1}(\chi)$ by $\Sigma_{1}^{1}(\chi ; A)$.

We now come to the proof of $(\mathrm{b})$. So assume $\mathfrak{F} \subseteq L(\mathbf{R}), \mathfrak{F}$ has cardinality $\leq 2^{\aleph_{0}}$ and $\mathrm{AD}(\mathfrak{F})$ holds. Then by (a) we surely have that at least $\mathrm{AD}^{L(\mathbf{R})}$ holds. In order to prove full $\mathrm{AD}$, consider an arbitrary game $A \subseteq \mathbf{R} \times \mathbf{R}$. Define then the following auxiliary game $A^{*} \subseteq \mathbf{R} \times \mathbf{R}$ :

$A^{*}(\sigma, \tau) \Leftrightarrow \sigma$ a strategy for $\mathrm{I} \wedge[\tau$ is a strategy for $\mathrm{II} \Rightarrow \sigma * \tau \in A]$,

where for strategies $\sigma, \tau$ for I, II, respectively, we let $\sigma * \tau$ be the outcome of the run of the game in which I follows $\sigma$ and II follows $\tau$. Thus $A^{*}$ is almost like $A$ except that players I and II play against each other's strategies instead of individual moves. (Strategies are of course viewed as reals.)

Now by our hypothesis there is $f \in \mathfrak{F}$ such that $\forall \tau(f(\tau), \tau) \in A^{*}$ or else there is $g \in \mathfrak{F}$ with $\forall \sigma(\sigma, g(\sigma)) \notin A^{*}$. The two cases are similar, so let us assume that we have the first one. Put

$$
B=\{f(\tau) * \tau: \tau \text { is strategy for II }\}
$$


Since $\forall \tau(f(\tau), \tau) \in A^{*}$, if $\tau$ is a strategy for II, then $f(\tau) * \tau \in A$, so $B \subseteq A$. But $f \in \mathfrak{F} \subseteq L(\mathbf{R})$, thus $B \in L(\mathbf{R})$, and so by $\mathrm{AD}^{L(\mathbf{R})}, B$ is determined. If I has a winning strategy in $B$ then I clearly has a winning strategy in $A$ and we are done; else II has a winning strategy $\tau_{0}$ in $B$. Let $\sigma_{0}=f\left(\tau_{0}\right)$. Then $\sigma_{0} * \tau_{0} \notin B$, but by definition of $B$ we also have $\sigma_{0} * \tau_{0} \in B$, a contradiction, and our proof is complete.

As a final illustration of this technique let us give a simple proof of Kunen's result that $\mathrm{AD}(\mathrm{Borel}) \Rightarrow \mathrm{AD}$.

Assume $\mathrm{AD}$ (Borel). Then it is easy to verify that we have hyperdegree determinacy, i.e., every set of hyperdegrees contains or is disjoint from a cone of hyperdegrees. From this it follows as usual that $\boldsymbol{\kappa}_{1}$ is measurable and thus $\boldsymbol{\Sigma}_{1^{1}}^{1}$ Determinacy holds (the arguments involved here are of course due to Martin for the case of Turing degrees, and carry over trivially to hyperdegrees). Let now $A \subseteq \mathbf{R} \times \mathbf{R}$ be any game and consider the game $A^{*}$ as before. Using $\operatorname{AD}$ (Borel), let us say that $f$ is Borel with $\forall \tau(f(\tau), \tau) \in A^{*}$. Form as before the set $B=\{f(\tau) * \tau: \tau$ is a strategy for II $\}$. Then $B \in \Sigma_{1}^{1}$, so $B$ is determined and the rest of the argument is exactly the same.

\section{REFERENCES}

1. A. Blass, Determinateness and continuity, Proc. Amer. Math. Soc. 37 (1973), 572-574.

2. H. Friedman, private communication, January 1975.

3. L. A. Harrington and A. S. Kechris, On the determinacy of games on ordinals, Ann. Math. Logic 20 (1981), 109-154.

4. A. S. Kechris and W. H. Woodin, The equivalence of partition properties and determinacy, Proc. Nat. Acad. Sci. U.S.A. 80 (1983), 1783-1786.

5. A. S. Kechris, E. M. Kleinberg, Y. N. Moschovakis and W. H. Woodin, The axiom of determinacy, strong partition properties and nonsingular measures, Cabal Seminar 77-79 (A. S. Kechris, D. A. Martin and Y. N. Moschovakis, eds.), Lecture Notes in Math., Vol. 839, Springer-Verlag, Berlin and New York, 1981.

6. K. Kunen, private communication, February 1975.

7. J. Mycielski, On the axiom of determinacy, Fund. Math. 53 (1964), 205-224.

Department of Mathematics, California institute of Technology, Pasadena, California 91125 